\title{
Surface Temperature Estimation of Li-ion Battery via Thermal Impulse Response Technique
}

\author{
Ying Xiao \\ Renewable Energy and Vehicular Technology Laboratory \\ University of Texas at Dallas, USA \\ ying.xiao@utdallas.edu
}

\author{
Dimitri Torregrossa, Mario Paolone \\ Distributed Electrical Systems Laboratory \\ Swiss Federal Institute of Technology, Switzerland \\ dimitri.torregrossa@epfl.ch,mario.paolone@epfl.ch
}

\begin{abstract}
This paper focuses on the prediction of temperature profiles on the surface of Lithium-ion cells. In particular, the paper proposes the adoption of the impulse response technique to predict cell surface temperatures consequent to generic discharge conditions applied to the targeted cell. The method is fed by data obtained by dedicated experiments to be performed on the targeted cell. In order to feed the proposed method, these experiments aim at obtaining cell surface temperature profiles consequent to short-time current discharge impulses. A set of dedicated validations is finally included in the paper in order to verify the validity of the proposed method.
\end{abstract}

Keywords - thermal modelling, temperature prediction, lithiumion battery, energy storage systems, impulse response.

\section{INTRODUCTION}

Due to their high energy/power density and relatively low self-discharge capability, Li-ion batteries have been intensively investigated and commercially employed in advanced electrified vehicles.

In electric vehicles, during specific operating conditions like fast acceleration and regenerative braking phases, high current magnitudes flow in the cells. Consequently, the heat generation is intensified and it involves fast temperature rises and uneven temperature distribution throughout the batteries [1]. In this respect, to maintain a safe and optimal operational condition in electric vehicles, the accurate prediction of the cell temperature distribution is crucial.

A comprehensive state of the art associated to model for temperature estimation of electrochemical batteries can be divided into two main groups: (i) numerical techniques [2]-[4] and (ii) lumped thermal models [5]-[7]. Numerical techniques, such as finite element analysis (FEA) methods, have been successfully applied to infer the temperature distribution of electrochemical cells of different sizes and materials [2]-[3]. However, even if these models might be very accurate, due to their computational burden, they cannot be used in real time battery management systems. Additionally, in order to obtain accurate results, it is fundamental to know the internal structure and material properties of the targeted cell. This information is, in general, not easily available and might also evolve during the cell ageing. On the other hands, lumped thermal models, do not require high computational efforts. This category of models has been successfully used to study either surface and core temperatures of cylindrical battery cells. Due to their inherent simplicity, these methods could be used for on board automotive temperature control (e.g., [6]). However, the main hypothesis assumed in this category of models is that temperature distribution is cylindrically uniform. This assumption introduces significant error in the case of pouch cells, as it has been insightfully described in [4]. Moreover, it would be interesting to predict the temperature distribution of the targeted cell under different State-of-Health (SOH) and associated ageing.

The concept of impulse response technique has been introduced in the electrochemical battery and electric motor applications [8]-[11]. The authors of [8]-[9] estimated the State-of-Charge (SOC) of the battery by measuring the terminal voltage of the battery induced by an impulse current. Various impulse responses at different SOC levels are captured and stored in dedicated look-up tables. The terminal voltage of the battery, for a known input current, can be computed by convolving the current with all the stored impulse responses (i.e., each impulse response is captured at a known temperature and at a known SOC). By comparing the computed voltages with respect to the measured ones using a given pattern recognition techniques [9], the proper impulse response related to the specific SOC would be determined.

A similar approach has been recently proposed in [10]-[11] to estimate the radial vibration in permanent-magnet synchronous machine (PMSM) [10] and acoustic noise in switched reluctance machine (SRM) [11] using the mechanical impulse response of the stator frame.

In this paper, the concept of impulse response is proposed to accurately predict the temperature variations on the surface of electrochemical cells. The main idea is to investigate in which condition the use of the convolution theorem for temperature prediction is correct since, by nature, the targeted system exhibit inherent nonlinear behaviour.

The paper is structured as follows. Section II describes the fundamental concept of impulse response and determines the thermal time constant of the targeted electrochemical cell. Section III illustrates the experimental validation of the proposed technique for thermal mapping on the targeted electrochemical cell. Conclusions summarizing the main results are in Section IV. A dedicated appendix illustrates the experimental setup used to feed and validate the proposed method. 


\section{FUNDAMENTALS OF IMPULSE RESPONSE CONCEPT AND EXPERIMENTALLY-OBSERVED THERMAL TIME CONSTANTS}

\section{A. Summary on the Impulse Response of Linear Systems}

Based on linear system theory, the zero state output $y[k]$ of a linear time invariant (LTI) system for an arbitrary input $x[k]$ can be determined using its impulse response $h[k]$ as follows:

$$
y[k]=x[k] * h[k]=\sum_{m=-\infty}^{+\infty} x[m] \cdot h[k-m]
$$

where $x[k], h[k]$ and $y[k]$ are the input, impulse response and output of the system, respectively. Based on this theory, the output of the system associated to any arbitrary time evolution of input can be computed by convolving the impulse response with the arbitrary input. It is important to underline that the time duration of the impulse (i.e. $\Delta$ ) used to obtain the impulse response should be sufficiently smaller than the shortest dynamic of the targeted system, namely its time constant $\tau$.

For the state of charge estimation of a Lithium-ion cell, the relationship between these two parameters has been investigated in [8] and the following inequality has been inferred in order to obtain satisfactory results:

$$
\tau / \Delta>18
$$

It is worth nothing that the amplitude of the impulse in (1) is unitary. Concerning the best magnitude of the impulse, the criteria for its choice is based on the possibility to observe a measurable output variation. Therefore, if the system is assumed to be linear, it is possible to scale the output of the system to a unitary impulse.

\section{B. Experimental Observations of Lithium Cell Surface Thermal Time Constant}

The idea of the work here proposed is to experimentally investigate the relationship between impulses of discharge currents delivered by the targeted cell and its surface temperature variations. Fig. 1 summarizes the impulse response mapping, where $h_{D R}$ is the transfer function between electrical discharge current and surface temperature on its surface. In this respect, it is worth underlining that, since the internal battery resistance might vary during the application of the current impulse, there is the need to associate the evolution of the cell surface temperatures to its internal heat generation and this transfer function can be represented as $h_{I D R}$ as illustrated in Fig. 1.

The targeted battery, shown in Fig. 2(a), is a $\mathrm{LiFePO}_{4}$ battery with nominal capacity of $70 \mathrm{Ah}$ and nominal voltage of $3.3 \mathrm{~V}$. This cell has a specific energy density of $100 \mathrm{Wh} / \mathrm{kg}$. The expected lifetime declared by the manufacture is of 3000 cycles with a depth of discharge (DOD) of $70 \%$ within the voltage range of 2.5-3.6 $\mathrm{V}$. The admissible current range is up to $1 \mathrm{C}(70 \mathrm{~A})$ for charge and $3 \mathrm{C}(210 \mathrm{~A})$ for discharge.

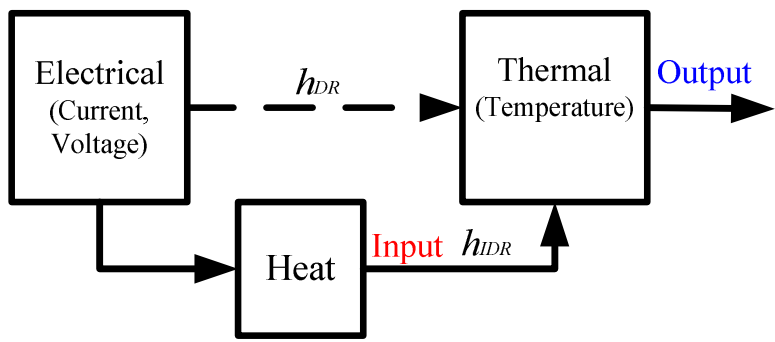

Fig. 1. Impulse response mapping the temperature prediction.

Fig. 2(b) illustrates the spatial location of the 24 temperature sensors used for the surface temperature measurements. In what follows, we represent the time evolution of the $N$-th temperature sensor $\left(T_{N}\right)$. Fig. 3(a) illustrates the time evolution of the average surface temperatures captured with all the available sensors $\left(T_{A V G}\right)$ during a discharge pulse at constant current (70 A) and at a constant ambient temperature $\left(22^{\circ} \mathrm{C}\right)$. The duration of the pulse is of $600 \mathrm{~s}$.

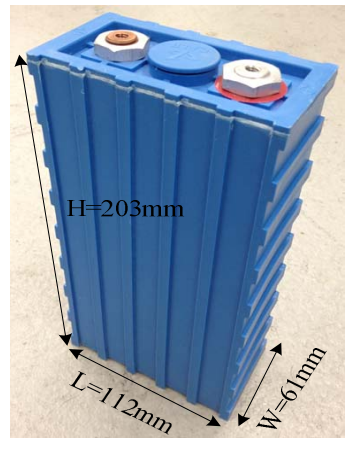

(a)

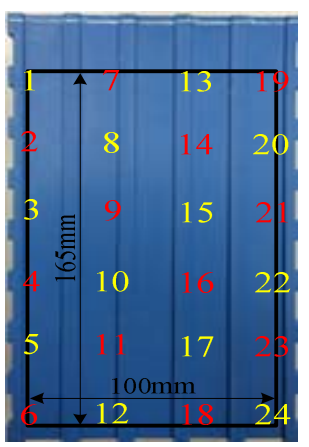

(b)
Fig. 2. (a) Targeted $\mathrm{LiFePO}_{4}$ battery and (b) location of temperature sensors (red locations refer to the front surface and yellow ones to the back surface).
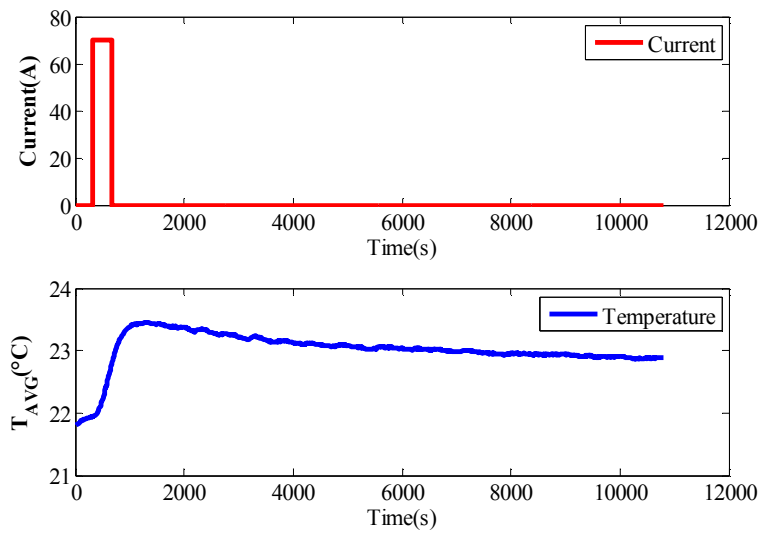

(a) 


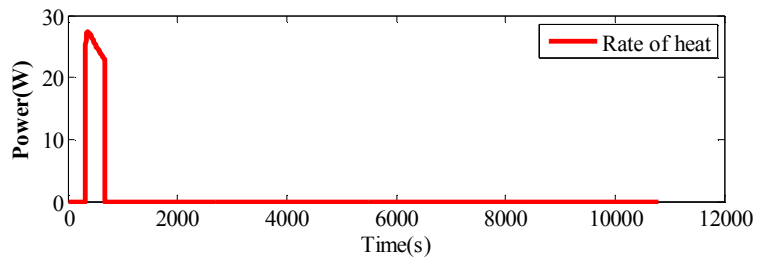

(b)

Fig. 3. (a) Current impulse and average cell surface temperature profile, (b) computed rate of heat.

In order to illustrate the results of Fig. 3(b), we here define the rate of heat generation computed as [12]:

$$
\dot{Q}=i\left\{V_{O C}(D O D)-V_{C E L L}(D O D)\right\}
$$

where $i$ is the current delivered by the cell, $V_{O C}(D O D)$ and $V_{C E L L}(D O D)$ are the open circuit voltage and the terminal voltage as a function of the depth of discharge (DOD), respectively. Fig. 3(b) illustrates the computed rate of heat using (3). The adopted values of $V_{O C}(D O D)$ and $V_{C E L L}(D O D)$ to obtain the results of Fig. 3(b) are given by Fig. 4 that illustrates the evolution of $V_{C E L L}$ and $V_{O C}$ for different DODs. The discharge current used to obtain these results is of $70 \mathrm{~A}$. The targeted cell, as shown in Fig. 2(a), has been kept into a climatic chamber at $25^{\circ} \mathrm{C}$.

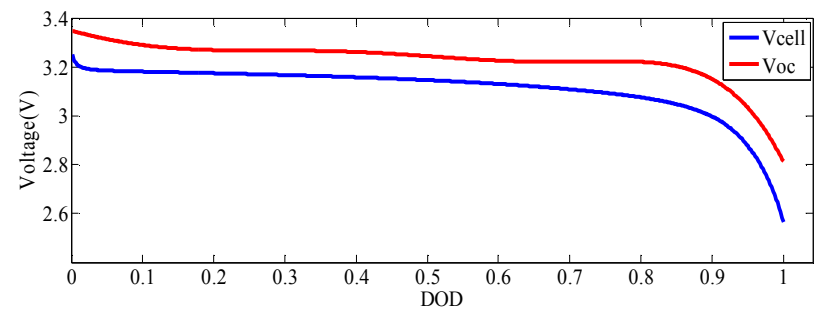

Fig. 4. $V_{\mathrm{CELL}}$ and $V_{O C}$ as a function of DOD (70 A constant discharge current).

\section{Thermal Time Constant Assessment}

In order to determine the thermal time constant of the system, a narrow pulse of current is delivered by the targeted cell and the corresponding voltage variation is measured to calculate the rate of heat. The temperatures of 24 targeted points located on the surface of the battery, shown in Fig. 2(b), are captured as well.

The same current magnitude of $70 \mathrm{~A}$ with three different durations, 1 minute, 6 minutes and 10 minutes, are applied to the battery and the corresponding rates of heat are considered as the impulses of the system. The temperature differences between the average battery surface and the ambient one are computed and considered as impulse responses (see Fig. 5).

As presented in Fig. 5(a), the rate of heat associated with 1 minute pulse is not enough to introduce significant temperature variation on the battery surface and therefore this heat pulse of 1 minute cannot be utilized as impulse of the battery system. Therefore, longer durations of 6 and 10 minutes are evaluated next to determine the proper width of the impulse satisfying (2).
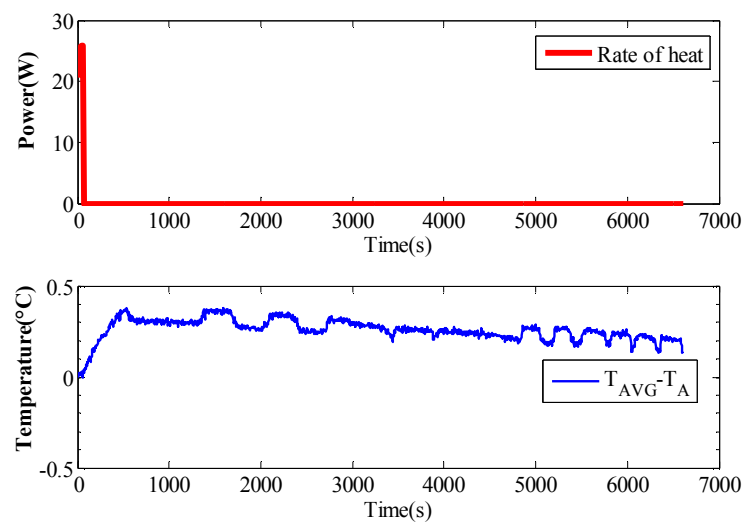

(a)
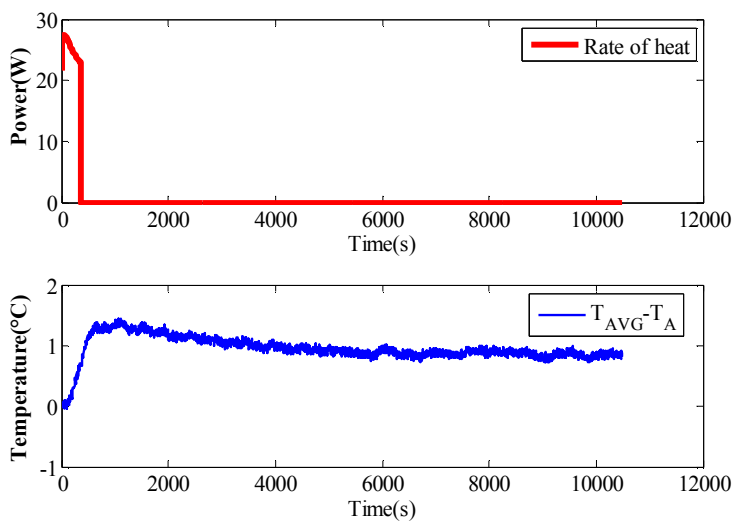

(b)
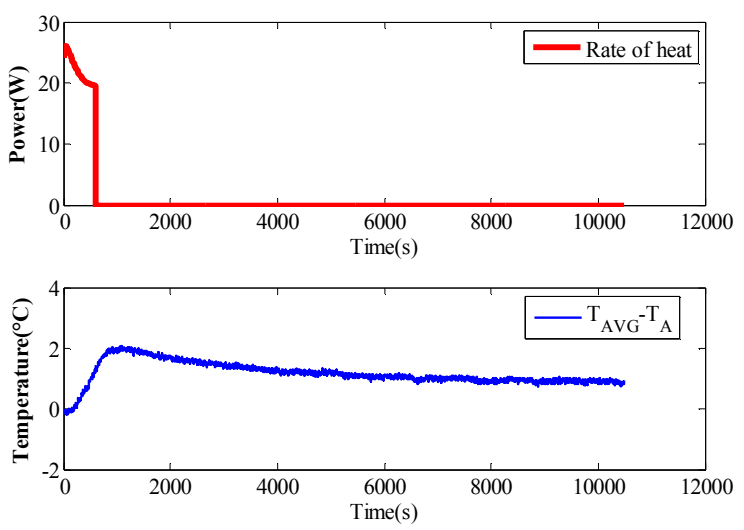

(c)

Fig. 5. Rates of heat impulse for applied current of (a) 1 minute (b) 6 minutes (c) 10 minutes and corresponding differences between the average cell surface temperature and the ambient one.

In order to investigate the value of time constant of the temperature variation evolution the difference $\Delta T$ between the $T_{A V G}$ and the ambient temperature $T_{A}$ has been measured 
for the two cases shown in Fig. 5, 6 minutes impulse and 10 minutes impulse. We have assumed a first order behaviour of the time evolution of $\Delta T$ :

$$
\Delta T(t)=\Delta T_{M A X} e^{-t / \tau}
$$

where $\Delta T_{M A X}$ is the maximum value of $\Delta T$.

By taking into account the measurement shown in Fig. 5(b) and (c), we found a time constant for the 6 minutes impulse equal to $9725 \mathrm{~s}$ and of $7854 \mathrm{~s}$ for the 10 minutes impulse. We can note that only the 6 minutes impulse satisfies equation (2) and, consequently, 6 minutes will be considered as a reference width for the thermal impulse.

\section{TEMPERATURE ESTIMATION METHOD}

\section{A. Thermal Impulse Response Measurements}

The surface temperatures of a cell experience variations in correspondence of a heat impulse due to the finite thermal capacity of the system.

The impulse responses measured in correspondence of the multiple surface points associated to the application of the $70 \mathrm{~A} / 6$ minutes current discharge profile are recorded. We can define individual impulse responses for each temperature sensor (i.e., $P_{1}, P_{2}$ and $P_{\mathrm{N}}$ ). The number of samples associated to each impulse response is $M$. During this measurement the targeted cell is located inside the climatic chamber at a known ambient temperature.

Fig. 6(a) illustrates the time evolution of the temperature impulse responses of some selected measurement points. $T_{A}$ is the ambient temperature during the experiment that, as it can be seen, is not constant during the test. The variations are due to the system control of the targeted climatic chamber (see the Appendix for further information about the climatic chamber characteristics). Fig. 6(b) illustrates the time evolution of the difference between the temperature of the above selected measurement points and $T_{A}$.

\section{B. Proposed Algorithm}

As previously explained, the $70 \mathrm{~A} / 6$ minutes discharge is considered to be the reference thermal impulse. Indeed, with respect to this current discharge profile, there is an associated thermal heat rate generated inside the targeted cell evaluated with (3). We henceforth define this heat rate as the reference one: $\dot{Q}_{R E F}$.

The idea of the proposed algorithm is to evaluate the temperature distribution of the targeted cell for generic discharge profiles.

The steps that compose the proposed algorithm are described below.

- Measure the cell current and the voltage evolutions associated to the generic current discharge profile (these quantities can be also inferred from a given cell electrical model).
- Evaluate the discrete time series of the heat rate associated to the targeted discharge current profile $\dot{Q}_{i}$ with (3). In particular, the discrete heat rate $\dot{Q}_{i}$ needs to be scaled with respect to $\dot{Q}_{R E F}$ and its relevant duration $T_{D}$ :

$$
\dot{Q}_{i R E F}=\frac{\dot{Q}_{i}}{\dot{Q}_{R E F}} \frac{T_{S}}{T_{D}}
$$

where, $T_{S}$ and $T_{D}$ are the sampling time $(5 \mathrm{~s})$ and the duration of the impulse $\dot{Q}_{R E F}$ (6 minutes).

- Convolve the $\dot{Q}_{i R E F}$ with the recorded $P_{i}$ thermal impulse response in order to evaluate the temperature of the targeted points $T_{i}$, as follows:

$$
T_{i}[k]=\dot{Q}_{i R E F} * P_{i}[k]=\sum_{m=0}^{M} \dot{Q}_{i R E F}[m] \cdot P_{i}[k-m]
$$

Fig. 7 illustrates the block diagram of the proposed method.

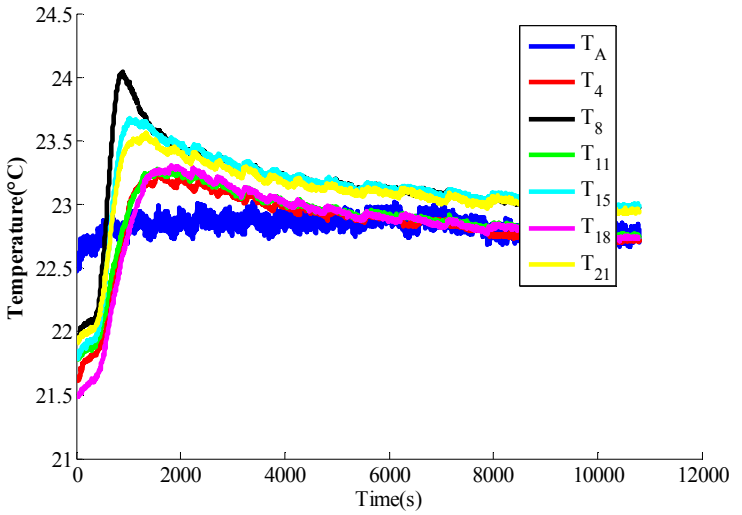

(a)

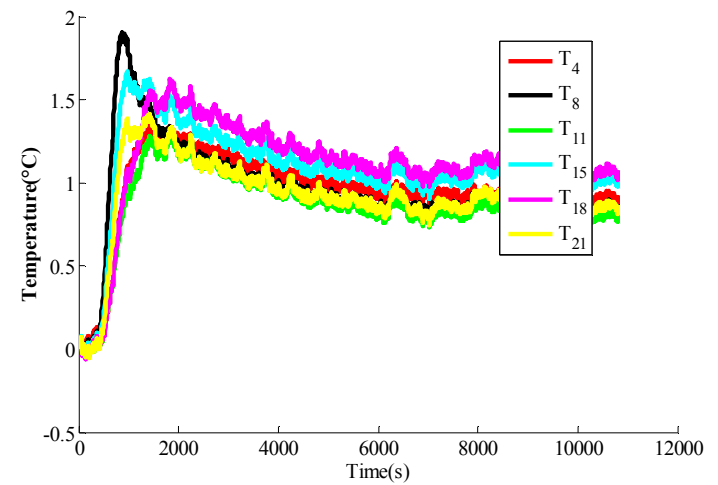

(b)

Fig. 6. (a) Impulse responses and (b) temperature variations at different points corresponding to reference heat impulse (70 A discharge current applied for 6 minutes). 


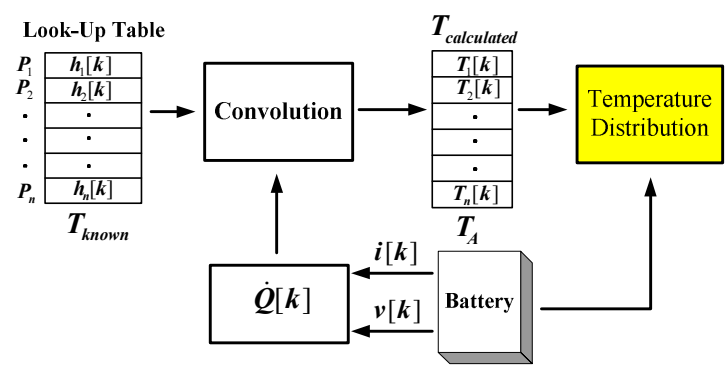

Fig. 7. Block diagram of the proposed method.

\section{EXPERIMENTAL VERIFICATION}

The aim of this section is to illustrate the experimental validation and the limit of the proposed algorithm. Table I summarizes the experimental tests carried out.

The experiment 1 is used as reference impulse response. The experiment 2 has a discharge current of $60 \mathrm{~A}$ for the first 3 minutes and $80 \mathrm{~A}$ for the following 3 minutes, the experiment 3 has a current profile of 30 A for first 4 minutes, $35 \mathrm{~A}$ for next 4 minutes, $50 \mathrm{~A}$ for another 2 minutes, and $30 \mathrm{~A}$ for the last 2 minutes. These two last experiments have a current with the same root mean square (RMS) value of the experiment 1 (the reference one).

TABLE I: LIST OF EXPERIMENTS FOR INPUT-OUTPUT LINEARITY

\begin{tabular}{|c|c|}
\hline Experiment & Current Profile \\
\hline 1 & 70 A for 6 minutes \\
\hline 2 & $60-80$ A for $3-3$ minutes \\
\hline 3 & $30-35-50-30$ A for $4-4-2-2$ minutes \\
\hline
\end{tabular}

Fig. 8-11 illustrate the measured temperature increase for point $T_{8}, T_{11}, T_{21}, T_{23}$ between the baseline impulse (70 A for 6 minutes) and experiment 2 (60-80 A for 6 minutes). Fig. 12-15 illustrate the same type of comparisons for the same measurement points and experiment 3. It is important to observe that even if the RMS value of the current is the same for the compared experiments, the temperature variations associated with the two current profiles are different. This is due to the fact that the internal losses, and the associated heat rates of the cell, are different for the various discharge profiles.

By observing the above figures, it is possible to make the following considerations:

- two main sub-phases could be detected, one associated to the temperature increase phase, and the other one associated to the temperature decrease subsequent to the interruption of the cell internal heat generation;

- during the temperature increase the matching between measurement and convolution result is very good, with an error lower than $2 \%$ (the reference temperature is $\left.T_{A}+\Delta T\right)$;

- during the temperature decrease, the matching is less accurate with errors in the order of $12 \%$.
Based on these considerations, it is possible to conclude that the proposed method provides consistent results to estimate the cell surface temperatures during the phases where the internal heat generation takes place (i.e., when the temperatures exhibit positive time derivatives). Indeed, even in presence of current profiles characterised by different durations or different magnitudes than those employed for the thermal reference impulse response, the proposed method is sufficiently accurate. On the contrary, during phases subsequent to the interruption of the cell internal heat generation, increased errors take place since the forcing function required by (6) is null and, therefore, the temperatures evolutions are dominated by the climatic chamber behaviour.

Concerning the computational requirements, it is worth mentioning that, on a platform comprising of a laptop with 2.4 $\mathrm{GHz}$ of CPU speed and 4GB of RAM, the above computations takes a negligible computation time.
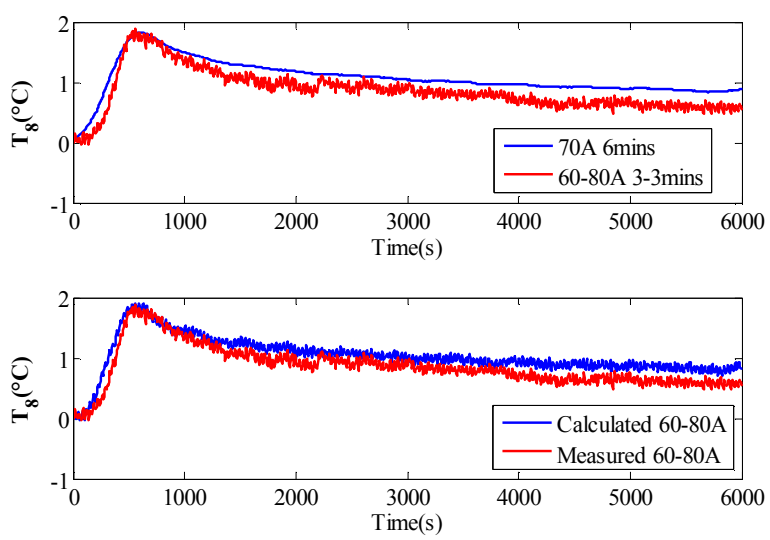

Fig. 8. Comparison of (a) original measurements and (b) convolution results for experiment 2 at the measurement point $T_{8}$ (maximum error is $1 \%$ during rising temperature phase).
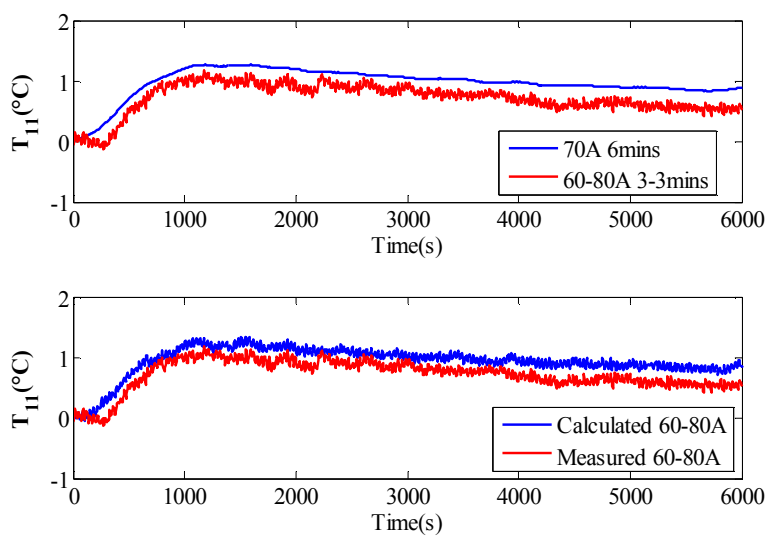

Fig. 9. Comparison of (a) original measurements and (b) convolution results for experiment 2 at the measurement point $T_{11}$ (maximum error is $2 \%$ during rising temperature phase). 

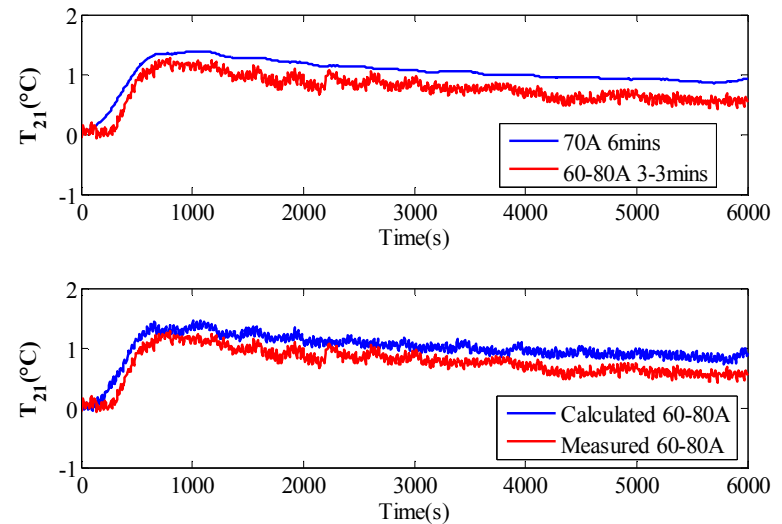

Fig. 10. Comparison of (a) original measurements and (b) convolution results for experiment 2 at the measurement point $T_{21}$ (maximum error is $1.75 \%$ during rising temperature phase)
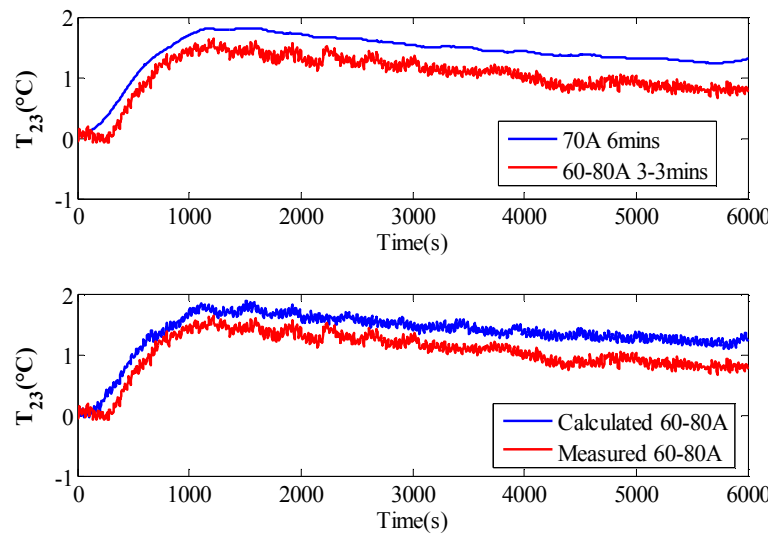

Fig. 11. Comparison of (a) original measurements and (b) convolution results for experiment 2 at the measurement point $T_{23}$ (maximum error is $1.5 \%$ during rising temperature phase)
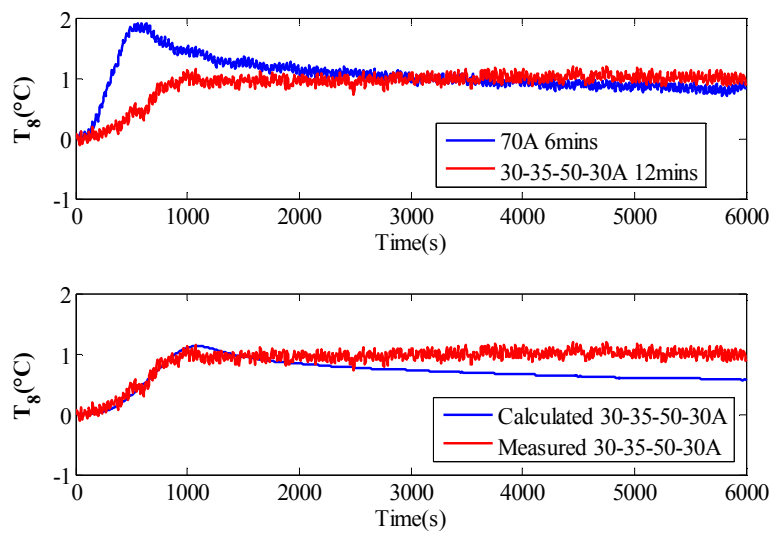

Fig. 12. Comparison of (a) original measurements and (b) convolution results for experiment 3 at the measurement point $T_{8}$ (maximum error is $1.6 \%$ during rising temperature phase).
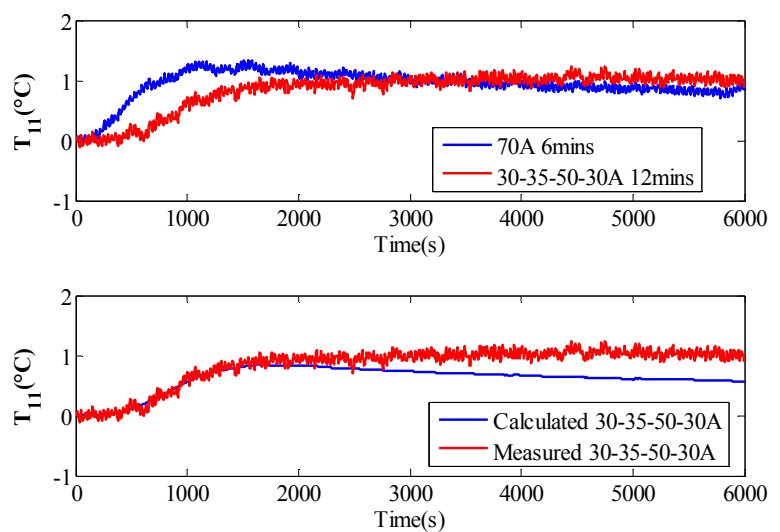

Fig. 13. Comparison of (a) original measurements and (b) convolution results for experiment 3 at the measurement point $T_{11}$ (maximum error is $1 \%$ during rising temperature phase).
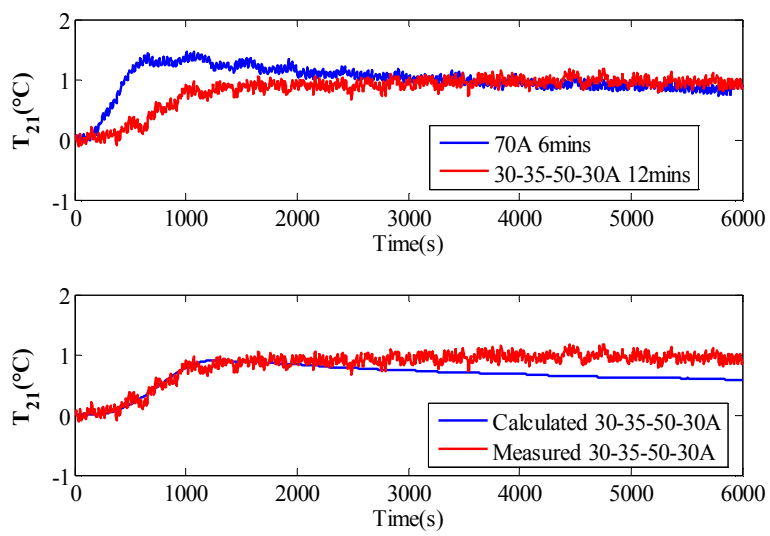

Fig. 14. Comparison of (a) original measurements and (b) convolution results for experiment 3 at the measurement point $T_{21}$ (maximum error is $1 \%$ during rising temperature phase).
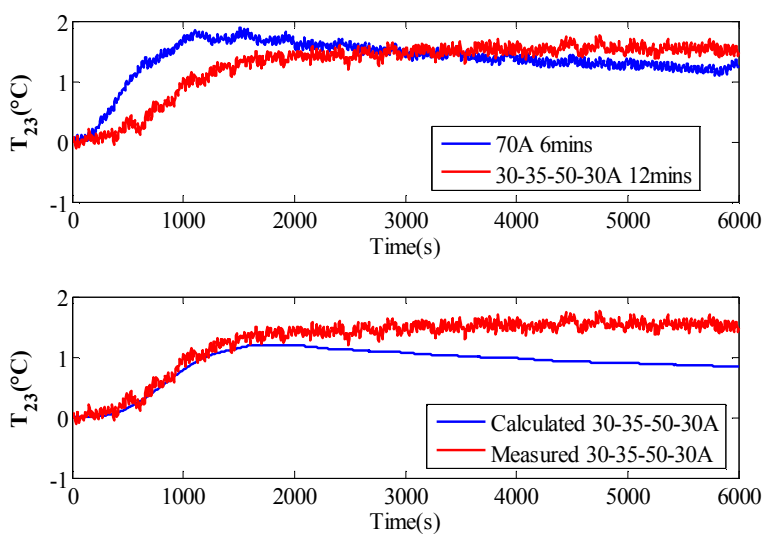

Fig. 15. Comparison of (a) original measurements and (b) convolution results for experiment 3 at the measurement point $T_{23}$ (maximum error is $1 \%$ during rising temperature phase). 


\section{CONCLUSIONS}

In this paper, a temperature prediction method based on impulse response technique for Li-ion battery has been presented.

Experimental investigations have been allowed to determine the width and the magnitude of the current to infer a reference thermal impulse. This thermal impulse has been used to obtain the associated impulse responses of a given number (24) of temperature measurements placed on the targeted cell surfaces. The convolution theorem is used to compute the temperature variations associated to current discharge profile having duration and magnitude other than that used for the impulse reference response.

The experimental results have shown a very good agreement between measurements and temperatures predicted by the proposed algorithm. The proposed method is able to accurate track the temperature evolution during its rising phase. The associate maximum error is lower than $2 \%$.

Further outlooks of the proposed method will focus on its validation with respect to generic cell state-of-charge. Indeed, different values of this quantity largely affect the linearized response of the system under study.

\section{APPENDIX}

The experimental setup shown in Fig. 16 is composed of following devices:

1) A TestEquity $115 \mathrm{~A}$ model climatic chamber characterised by an operating temperature range of $-73{ }^{\circ} \mathrm{C}$ to $+175^{\circ} \mathrm{C}$ with a ripple of $\pm 1.0^{\circ} \mathrm{C}$ (in steady state).

2) $25 \mathrm{~J}$-type thermocouples with temperature measurement range from 0 up to $750{ }^{\circ} \mathrm{C}$, maximum error of $\pm 2.2{ }^{\circ} \mathrm{C}$. The thermocouples are located on the cell surface as shown in Fig. 2(b). An additional sensor is used to measure the ambient temperature in the chamber.

3) A Magna-Power XR50-80 series power supply and a Chroma 63203 electronic load are used to charge and discharge the cell. The power supply has a nominal power of 4 $\mathrm{kW}$ with maximum voltage and current of $50 \mathrm{~V}$ and $80 \mathrm{~A}$. The nominal power of the electronic load is $5.2 \mathrm{~kW}$ with maximum voltage and current of $80 \mathrm{~V}$ and $600 \mathrm{~A}$.

4) An Agilent 34972A, interfaced with a personal computer, has been adopted to digitize voltage, current and temperature measurements. The sampling time is of $5 \mathrm{~s}$ for the temperature and $50 \mathrm{~ms}$ for current and voltage.

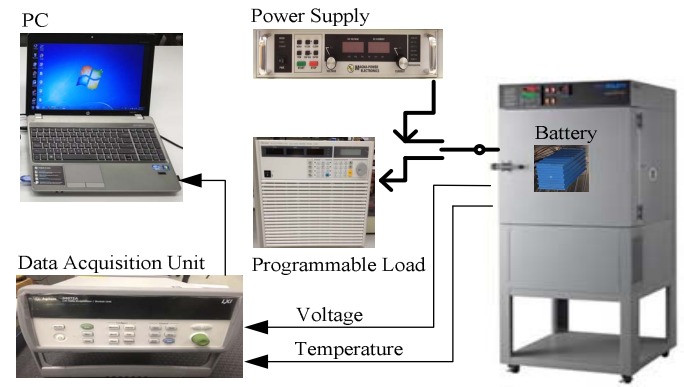

Fig. 16. Schematic representation of the experimental bench-test.

\section{ACKNOWLEDGMENT}

The authors would like to thank Professor Babak Fahimi, the director of Renewable Energy and Vehicular Technology lab at University of Texas at Dallas. He provided, along with his scientific advice, all the equipment for the experimental investigations illustrated in the paper.

The authors thankfully acknowledge the Swiss Innovation and Technology Committee (CTI) since the scientific activity described in this paper is framed within the context of the researches of the Swiss Competence Center for Energy Research "FURIES" (Future Swiss Electrical Infrastructure).

\section{REFERENCES}

[1] X. Hu, L. Chaudhari, S.H. Lin, S. Stanton, S. Asgari, and W.Y. Lian, "A State Space Thermal Model for HEV/EV Battery Using Vector Fitting," in Proc. IEEE ITEC, 2012, pp. 1-8.

[2] Y. Inui, Y. Kobayashi, Y. Watanabe, Y. Watase, and Y. Kitamura, "Simulation of temperature distribution in cylindrical and prismatic lithium ion secondary batteries," J. Ener. Conv. and Manag., vol. 48, Issue.7, pp. 2103-2109, 2007.

[3] C. Forgez, D. V. Do, G. Friedrich, M. Mocrette, and C. Delacourt, "Thermal modeling of a cylindrical $\mathrm{LiFePO}_{4} /$ graphite lithium-ion battery," J. of Power Sources, vol. 195, no. 9, pp. 2961-2968, Nov. 2009.

[4] K. Murashko, J. Pyrhönen and L. Laurila, "Three-Dimensional Thermal Model of a Lithium Ion Battery for Hybrid Mobile Working Machines: Determination of the Model Parameters in a Pouch Cell," IEEE Trans. Energy Convers., vol. 28, no. 2,pp. 335-343, 2013.

[5] I. Arasaratnam, J. Tjongy, R. Ahmed, M. El-Sayed and S. Habibi, "Adaptive Temperature Monitoring for Battery Thermal Management," in Proc. IEEE ITEC, 2012, pp. 1-6.

[6] X. Lin, H. E. Perez, J. B. Siegel, A. G. Stefanopoulou, Y. Li, R. D. Anderson, Y. Ding, and Castanier, M.P, "Online Parameterization of Lumped Thermal Dynamics in Cylindrical Lithium Ion Batteries for Core Temperature Estimation and Health Monitoring," IEEE Trans. Cont. Sys. Tech., vol. 21, no. 5, pp. 1745-1755, Sep. 2013.

[7] Y. Kim; S. Mohan; J.B. Siegel; A.G. Stefanopoulou, and Y. Ding, " The Estimation of Temperature Distribution in Cylindrical Battery Cells Under Unknown Cooling Conditions," IEEE Trans. Cont. Sys. Tech., no. 99, pp. 1-10, Mar. 2014.

[8] A. H. Ranjbar, A. Banaei, A. Khoobroo, B. Fahimi, "Online Estimation of State of Charge in Li-Ion Batteries Using Impulse Response Concept", IEEE Trans. Smart Grid, vol.3, Issue 1, pp. 360-367, 2012.

[9] Y. Xiao, C. Lin, and B. Fahimi, "Online State of Charge Estimation in Electrochemical Batteries: Application of Pattern Recognition Techniques," in Proc. IEEE APEC, pp.2474-2478, 2013.

[10] D. Torregrossa, B. Fahimi, F., Peyraut, and A., Miraoui, "Fast Computation of Electromagnetic Vibrations in Electrical Machines via Field Reconstruction Method and Knowledge of Mechanical Impulse Response," IEEE Trans. Ind. Electron., vol.59, no.2, pp.839-847, Feb. 2012.

[11] C. Lin, and B. Fahimi, "Prediction of Acoustic Noise in Switched Reluctance Motor Drives", IEEE Trans. Energy Convers., vol.28, no.1, pp.250-258, Mar. 2014.

[12] Y. Xiao, "Model-Based Virtual Thermal Sensors for Lithium-ion Battery in EV Applications," in IEEE Trans. Ind. Electron., 2015. 